\title{
Influence de la fréquence de distribution et de la forme de présentation d'un foin de luzerne sur les temps de rétention moyens de différents marqueurs du transit digestif chez le mouton
}

\author{
A de Vega, J Gasa, C Castrillo, J Balcells, JA Guada \\ Universitad de Zaragoza, facultad de veterinaria, departamento de producción animal y \\ ciencia de los Alimentos, Miguel Servet 177 50013, Saragosse, Espagne
}

\begin{abstract}
Summary - Effect of feeding frequency of low quality lucerne hay offered chopped or ground and pelleted on markers' total mean retention time. Feeding chopped or ground and pelleted lucerne hay twice a day ad libitum or 12 times a day did not affect markers' total meen retention time (TMRT) measured in faeces, but increased it when eating time was limited to $3 \mathrm{~h} / \mathrm{d}$, possibly due to lower dry matter intake. Physical processing decreased TMRT of CoEDTA and showed a strong tendency to reduce it with ytterbium labelled on hay.
\end{abstract}

Les modèles mathématiques employés pour estimer le temps de rétention moyen d'un marqueur dans le tube digestif à partir de l'évolution de sa concentration dans les fèces supposent l'existence de conditions d'état stable. De tous les facteurs qui ont une influence sur le maintien de ces conditions, la fréquence de distribution de l'aliment est considérée comme l'un des plus importants. L'objectif de ce travail a été d'étudier chez le mouton l'effet de la fréquence journalière de distribution d'un foin de luzerne offert, haché ou broyé et aggloméré sur l'ingestion de matière sèche, la digestibilité de la matière organique et le temps de rétention moyen des marqueurs dans le tractus digestif.

Matériel et méthodes - Deux lots de 6 agnelles âgées de 11 mois et d'un poids vif moyen de $34,4 \pm 0,4 \mathrm{~kg}$ ont reçu du foin de luzerne haché $(H)$ ou broyé $(2 \mathrm{~mm})$ et granulé $(G)$. L'aliment a été distribué successi- vement selon 3 fréquences différentes: une fois par jour, avec un accès de $3 \mathrm{~h}$ (1D), 2 fois par $\mathrm{j}$, ad libitum (2D), et 12 fois par jour et une ingestion limitée à $90 \%(\mathrm{H})$ et $95 \%$ (G) de celle estimée dans la période précédente (12D) pour éviter les refus à la fin de chaque distribution. Dans chaque période, l'ingestion journalière de matière sèche (MSI) et la digestibilité de la matière organique $(\mathrm{dMO})$ des aliments ont été mesurées, puis des doses uniques de CoEDTA en solution aqueuse ( $35 \mathrm{mg}$ Co dans $50 \mathrm{ml}$ d'eau) et d'aliment mordancé au $\mathrm{Cr}$ (10 g/dose; $5 \mathrm{mg} \mathrm{Cr} / \mathrm{g}$ ) ou marqué avec de l'Yb (10 g/dose; $1 \mathrm{mg} \mathrm{Yb/g}$ ) ont été administrées simultanément par voie buccale. Le CoEDTA et l'aliment mordancé ont été préparés par la technique d'Uden et al (1980) et l'ytterbium fixé par immersion de l'aliment pendant $12 \mathrm{~h}$ dans une solution de $\mathrm{pH} 6,0$, à raison de $17 \mathrm{~g}$ d'acétate d'ytterbium par $\mathrm{kg}$ de MS d'aliment. Des prélèvements de fèces ont été effectués par collecte totale toutes les $3 \mathrm{~h}$ pendant les premières $24 \mathrm{~h}$ après l'administration des marqueurs, puis à $28,33,39,48$, $60,72,96,120$ et $144 \mathrm{~h}$. Les marqueurs ont été dosés dans les fèces par spectrométrie d'émission par plasma, et les résultats ajus- 
Tableau I. Effet de la forme de présentation ( $H$ vs $G$ ) et de la fréquence de distribution (1D, 2D et 12D) sur l'ingestion de matière sèche (MSI, $\mathrm{g} / \mathrm{kg} \mathrm{PV}^{0.75}$ ), la digestibilité de la matière organique (dMO, \%) et les temps de rétention moyen TRM, h) dans le tube digestif de CoEDTA et des aliments marqués avec le $\mathrm{Cr}$ et l'Yb.

\begin{tabular}{|c|c|c|c|c|c|c|c|c|}
\hline & \multirow[b]{2}{*}{$H$} & \multirow[b]{2}{*}{$G$} & \multirow[b]{2}{*}{$1 D$} & \multirow[b]{2}{*}{$2 D$} & \multirow[b]{2}{*}{$12 D$} & \multirow[b]{2}{*}{ ETM } & \multicolumn{2}{|c|}{ Effet } \\
\hline & & & & & & & Prés & Freq \\
\hline MSI & 64,2 & 87,3 & 57,4 & 88,6 & 81,3 & 5,17 & 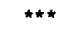 & $\star \star \star *$ \\
\hline $\mathrm{dMO}$ & 53,2 & 49,1 & 51,0 & 51,7 & 50,8 & 0,009 & $* \star *$ & ns \\
\hline TRM Cr & 45,8 & 45,5 & 60,2 & 39,5 & 37,4 & 2,417 & ns & $* * *$ \\
\hline $\mathrm{Yb}$ & 40,0 & 34,5 & 46,2 & 32,0 & 33,9 & 2,570 & ns & *** \\
\hline Co & 30,5 & 25,3 & 35,2 & 23,5 & 25,0 & 1,498 & $\star$ & 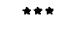 \\
\hline
\end{tabular}

tés au modèle de Dhano et al (1985) pour obtenir les temps de rétention moyens (TRM).

Résultats et discussion - Les principaux résultats sont rassemblés dans le tableau I. La MSI a été inférieure avec le traitement $1 \mathrm{D}$ et c'est avec ce rythme d'alimentation que le TRM des 3 marqueurs a été le plus grand. Pourtant, la fréquence de distribution n'a pas affecté la $\mathrm{dMO}$, ce qui suggère une réduction de l'efficacité des processus digestifs et en particulier de la fermentation microbienne dans le rumen avec le traitement 1D. II n'y a pas eu de différences dans les TRM des 3 marqueurs entre $2 D$ et $12 D$, malgré l'observation de comportements alimentaires très différents. La fréquence d'alimentation n'a pas eu ou a eu très peu d'effets sur la validité d'ajustement des points au modèle de Dhanoa et al (1985) qui a expliqué, dans tous les cas, plus de $90 \%$ de la variance totale. Le broyage et l'agglomération du foin ont permis une plus grande $\mathrm{MSI}$ et ont réduit la $\mathrm{dMO}$. Cette forme de présentation n'a pas affecté le TRM du $\mathrm{Cr}$, a eu tendance à diminuer celui de $\mathrm{I} Y \mathrm{Yb}$ $(P<0,12)$ et a diminué de façon signi- ficative celui du CoEDTA. En accord avec Thomson et Beever (1980), les résultats montrent que la plus grande MSI enregistrée avec le foin $G$ par comparaison avec le foin $H$, associée à une dMO plus faible, peut être expliquée par une réduction du TRM de l'aliment dans le tube digestif, en particulier des particules véhiculées par la phase liquide.

En conclusion, l'absence de différences entre $2 D$ et $12 D$ suggère que les différences dans les conditions d'équilibre provoquées par ces 2 fréquences ne changent pas significativement le TRM des marqueurs de phase solide ou liquide dans le tube digestif. Le broyage et agglomération du foin réduit le TRM de l'Yb et du CoEDTA, bien que l'effet ne soit apparu significatif qu'avec ce dernier.

Dhanoa MS, Siddons RC, France J, Gale DL (1985) Br J Nutr 53, 663-671

Thomson DJ, Beever DE (1980) in : Digestive physiology and metabolism in the ruminant (Ruckebusch $Y$, Thivend $P$, eds) MTP Press Ltd

Uden P, Colluci E, Van Soest PJ (1980) J Sci Food Agric 31, 625-632 